\title{
Picture Browsing and Map Interaction using a Projector Phone
}

\author{
Andrew Greaves ${ }^{1}$, Alina Hang ${ }^{2}$, Enrico Rukzio ${ }^{1}$ \\ ${ }^{1}$ Computing Department, Lancaster University, UK, \{greaves, rukzio\}@comp.lancs.ac.uk \\ ${ }^{2}$ Media Informatics group, University of Munich, Germany, alina.hang@campus.Imu.de
}

\begin{abstract}
It is expected that projector phones (mobile phones with built-in pico projectors) will hit the market by 2010 . Such phones provide a completely new way to display information and interaction techniques. The system presented in this paper allows the simulation of these projector phones as the real devices are not yet available. Through this, we demonstrate that it is currently possible to design, develop and evaluate applications for projector phones. The system supports three different modalities in order to compare when which display (phone display, projection) should be used. This prototype system was used for the implementation of two applications in order to test the advantages and disadvantages of projector phones for two common scenarios, picture browsing and map interaction. This demonstration paper describes the hardware used for the simulation of projector phones and the two developed prototypes. These prototypes were used for two different studies discussed in [1] and [2].
\end{abstract}

\section{Categories and Subject Descriptors}

H.5.2 [Information Interfaces and Presentation]: User Interfaces - Input devices and strategies; Prototyping.

\section{General Terms}

Design, Experimentation, Human Factors.

\section{Keywords}

Projector phone, photo browsing, map interaction.

\section{INTRODUCTION}

The limited screen size prevents the user from using the mobile phone to its full potential. The context and richness of information continues to increase with the development of new applications and so does the demand for this content to appear on mobile phones. While this information is presented in a high resolution, the problem is how to display this on such a small screen whilst still preserving the aesthetics and impact of the content.

Many have focused on different ways to overcome the small screen size of mobile devices. The typical approach is to zoom and scroll, however, the approach we see employed in classic desktop solutions falls short when deployed on a mobile device. Overview and detail, two screens are used, one for the overall view and one for a detailed view, and focus and context are e.g.

Permission to make digital or hard copies of all or part of this work for personal or classroom use is granted without fee provided that copies are not made or distributed for profit or commercial advantage and that copies bear this notice and the full citation on the first page. To copy otherwise, or republish, to post on servers or to redistribute to lists, requires prior specific permission and/or a fee.

MobileHCI 2008, September 2-5, 2008, Amsterdam, the Netherlands. Copyright (C) 2008 ACM 978-1-59593-952-4/08/09 ...\$5.00. two desktop PC approaches which do not work for mobile phones [3]. Many research projects have focused on solutions to address the issue of the small screen. Halo for instance is an interaction technique for interacting with maps that provides an indication of the existence of points of interest not currently shown on the display [4]. Interaction with big screens, as done by the Hermes Photo Display [5] and SharedNotes [6], is an alternative approach to solving the small screen problem however big screens are fixed in the environment, still costly, problems can arise with multiple users and they don't have the same mobile appeal and offer the same interactions provided by the mobile phone.

At present huge interest by Texas Instruments [7], Microvision in cooperation with Motorola [8], 3M [9] and Nokia [10] is being expressed in projectors that can be used in combination with or embedded into mobile phones and devices. Texas Instruments for instance unveiled a full working prototype of its Pico-Projector at CES 2008 [7]. It is expected that by 2010 consumers shall see these devices emerge in the market place $[8,10]$.

When projectors embedded into mobile devices such as PDAs and mobile phones become reality, the inherent display limitations of mobile phones perceived by many as the biggest problem could now be the smallest. Embedding pico projectors into mobile phones opens many doors; specifically it allows larger limitless displays to be dynamically created practically on any display surface. There is a whole new information space previously not even considered to now explore.

Typical interaction using a mobile phone requires the user to look at the mobile phones screen whilst at the same time looking at the device, specifically at the interaction controls provided. The integration of a projector into a mobile phone opens up two further interactions; interacting with the phone whilst looking at a projection and interacting using a combination of the mobile phone screen and the projection in parallel. In the latter case it would be expected that the two displays would contain different but application related content.

At Mobile HCI 2008 we present two prototype applications; Photo Browsing and Map Interaction that benefit from mobile projection and put the three modalities described above into practice using these two common scenarios. Photo Browsing is a very common activity associated with the mobile phone. The mobile phone is a highly ubiquitous device and the ability to quickly capture an unexpected moment and share it is highly appealing. However browsing photos on a mobile phone with such a small screen requires many panning, scrolling and zooming interactions, these of course are frustrating and highly undesirable. The second prototype application is Map Interaction as it is now more common that we see Google Maps and Tom Tom for example deployed on the mobile phone. Again map interaction 
using mobile devices requires many zooming, and scrolling actions when viewing a map on a small screen device.

\section{HARDWARE}

This section describes the hardware which can be used to simulate a projector phone and which was used to implement the three different interaction modalities phone display only, projection only and the combination of phone display and projection.

\section{Mobile Phone}

The Nokia N95 has a display resolution of 240 x 320 pixels, an aspect ratio of $3: 4$ and is used in portrait format. The N95 is a Nokia Series $603^{\text {rd }}$ edition handset, it was primarily chosen due to its large screen size when compared to other mobile phones and also has a large amount of program memory for application use. The N95 also has a large keypad and a joystick for easy navigation and selection actions.

\section{Projector}

The Samsung SP-P310ME is a battery powered hand held projector, coupled with a Nokia N95 it is used to simulate a projector phone. It has a relative size of $12.7 \times 9.5 \times 5.1 \mathrm{~cm}(\mathrm{w} \mathrm{x} \mathrm{d}$ $\mathrm{x}$ h) and weighs just 700 grams (excluding battery). The projector has a display resolution of $1027 \times 768$ pixels. Figure 1 illustrates the experimental setup. It can be expected that when using a projector phone the user will be unable to hold the mobile phone completely steady resulting in movement of the projection. For this reason the projector is attached to a frame using elastic to simulate realistic movement of the projection.
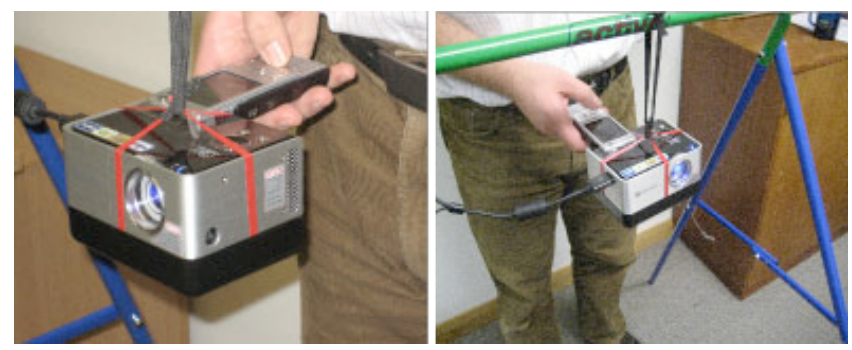

Figure 1. Prototype setup, N95 physically attached to a projector to simulate real use.

The N95 provides a TV-Out interface allowing it to directly connect to the projector using the Nokia TV Out cable (CA-75U) and the composite interface of the projector. Doing this, it is possible to directly project the contents of the mobile display. However, in doing so the N95 is unable to take advantage of the full resolution of the projector and the resulting projection is that of the mobile phone screen. Our approach involves connecting the projector to a laptop computer and projecting the current context of the laptop. When doing this the mobile phone is used to control the applications with the users looking at the projection.

In the projection only modality the display of the mobile phone is not used. Using the phone display and projection modality both displays (phone and projection) display information at the same time.

\section{PHOTO BROWSING}

A photo-browsing prototype was implemented to evaluate the three modalities when browsing photos.

\subsection{Picture Layout and Navigation}

For all three modalities (phone display only, projection only, and projection \& phone display) by default the images are displayed as thumbnails in a grid $3 \times 5$ displaying a maximum of 15 images at one time. A grid of these dimensions was chosen to favor and capture the most amount of content in relation to the mobile phone screen. Figure 2a illustrates the thumbnail layout for the mobile phone.

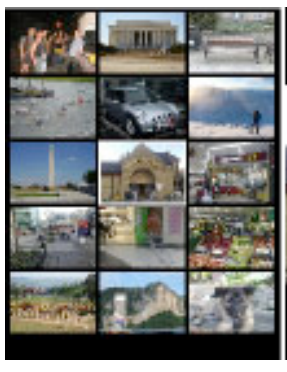

a

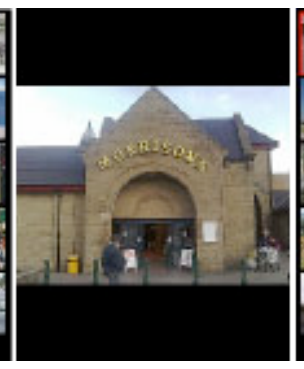

b

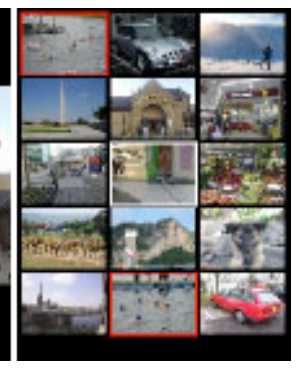

c
Figure 2. Mobile phone screen only image layout (a), enlarged image (b), multiple image selection (c).

The navigation structure coincides with standard navigation principles. This includes sequential browsing through the photos using the N95 joystick in all directions. Fast viewing shortcuts were also added; the user can quickly navigate to the last photo from the first photo and also navigate from the top row of photos to the bottom. A shift scrolling function was implemented to view the next photos within the collection that were not currently displayed. The shift scrolling function effectively reveals the next row of pictures when the use reaches the last displayed row with the top row disappearing. The shift function is slower and requires more browsing interactions when compared to an approach that would simply show the next 15 images. However, both the context and content are preserved allowing the use to still be aware of previous photos.

A white rectangle (Figure 2c) around the image identifies the current selected image. The user selects this image by pressing the ' 5 ' key on keypad. The selection is acknowledged by drawing a red square (Figure 2c) around the image. For all three modalities the user can enlarge an image which occupies the full width of the display. To keep the interaction as simple as possible it was decided that when an image is enlarged, navigation and selection actions are prohibited.

\subsection{Implementation of the Three Modalities Mobile Phone display only}

Figure $2 \mathrm{a}$ illustrates the photo browsing prototype using the mobile phone screen only. This is representative of typical interaction whilst browsing photos. The small screen size limits each photo to have a width of 74 pixels and a height of 55 pixels maintaining the correct $3: 4$ aspect ratio, a 4 pixel border separated adjacent images. Figure $2 b$ represents an enlarged image; the image occupies the full width of the display, 240 pixels with a resulting height of 180 pixels. The application was created for purposes of evaluation, as a result task time was sensitive and the delay in dynamically enlarging an image on the mobile phone could not be tolerated. For this reason each image is stored twice in its thumbnail and enlarged representation; doing so the images are enlarged with no delay. 


\section{Projection only}

Figure 3 illustrates the projection only interaction technique. The same characteristics as the mobile only technique apply, however, the photos are projected and the mobile screen is blank. The application is controlled using the mobile phone, the simplistic interaction controls used should enable the user to solely concentrate on the projection and control the application without needing to look down at the mobile phone. A ratio value between the height of the projector and mobile phone relative to the width of the mobile phone was calculated and applied to the projection. From the available projection size of 1024 x 768 pixels the ratio value yielded an application size with a width of 606 pixels and a height of 768 prior to projection.

At a projection distance of approximately $200 \mathrm{~cm}$ the maximum available projection size was a width of $100 \mathrm{~cm}$ by a height of $76 \mathrm{~cm}$. When obeying the ratio value, a projection of 606 by 768 pixels results in a total projection space of width $60 \mathrm{~cm}$ by a height of $76 \mathrm{~cm}$. Projecting the $3 \times 5$ thumbnail grid results in each thumbnail having a projection size of $20 \mathrm{~cm}$ by $15 \mathrm{~cm}$ and enlarged images having a size of $60 \mathrm{~cm}$ by $45 \mathrm{~cm}$.

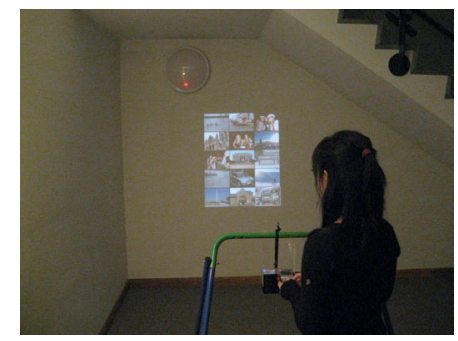

Figure 3. A user using the projection only interaction technique to browse pictures.

\section{Phone Display and Projection}

Figure 4 illustrates the phone and projection interaction technique. Here the user can use both displays in parallel to browse photos. The mobile phone displays the images using a thumbnail layout (Figure 4a) as with the mobile display only technique.

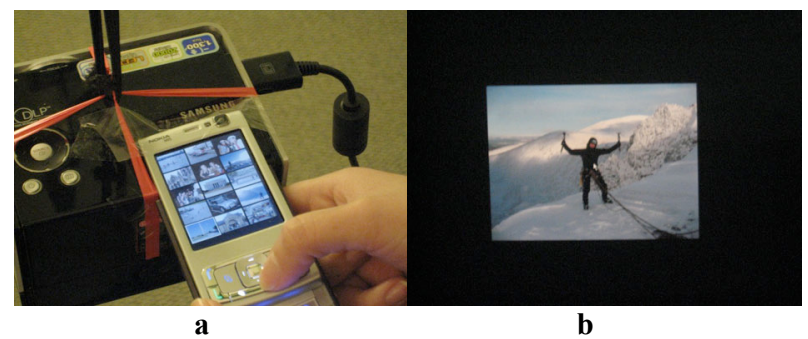

Figure 4. Phone and projection combination (a) thumbnail layout (b) User looking at an enlarged image in the projection.

The projection (Figure $4 \mathrm{~b}$ ) displays the currently selected image indicated on the mobile phone in a permanent enlarged view. The projection is automatically updated to reflect the currently selected picture on the mobile phone. It is still however possible to enlarge the images on the mobile phone. The user is free to choose the option that best suits them or the option that they feel most comfortable in using. Using the parallel approach allows the user to see instantly a bigger picture which may be the case for clarification if they are currently searching for a specific picture. The thumbnail view on the mobile phone preserves the current context of several images and allows the user to search among many pictures very quickly.

\subsection{Implementation}

\section{Mobile Phone}

Java ME (MIDP 2.0, CLDC 1.1) was used to implement the mobile phone application. The JSR-82 Bluetooth API was used to provide Bluetooth capabilities between the mobile phone and laptop computer. Due to the large number of photos and the program memory needed to store these photos during runtime it was not possible to store all the photos using the Record Store. All images were stored on a micro SD card and loaded into memory on demand using the JSR-75 File Connection API.

\section{Projection}

A Java SE application that represented the projection prototype as we see in Figures 3 and $4 \mathrm{~b}$ was created. A Bluetooth server application handled the communication between the laptop and mobile phone, on occurrence of every button press relevant to the application the button press id was sent over Bluetooth and interpreted, the relevant changes were then made to the projected content.

\section{MAP INTERACTION}

The second prototype is a map application that profits from the large high-resolution display of the projection. Similar to the photo browsing prototype the map interaction prototype was developed for the purpose of evaluation. The user had three tasks to complete; "Find the cheapest car park", "Select sights to visit" and "Find a restaurant" for each modality (phone display only, projection only, phone display and projection).

The projection interaction techniques provide distinct advantages with regards to the total displayable map area. Using the mobile phone a total area of 43750 square meters is visible on the map, $174 \mathrm{~m}$ in width and $250 \mathrm{~m}$ in height. The projection displays $370 \mathrm{~m}$ in width and $360 \mathrm{~m}$ in height resulting in a total displayable map area of 133200 square meters.

The N95 joystick allows the user to scroll the map in all four directions. Objects in the map could by selected by positioning the on screen cursor over the object and pressing the select button of the joystick. Map navigation and cursor movement were separate entities; keys 2, 4, 6 and 8 controlled the movement of the cursor.

\subsection{Find the cheapest car park}

Ten car park icons displaying hourly rates were randomly positioned around the map (see Figure 6a). The user has to navigate around the map and find and select the cheapest car park. Following selection the use is informed if the cheapest car park was found.

\subsection{Select Sights to visit}

For this task the user is presented with a map of a specific area surrounded by eleven different sights of interest to visit. Figure $6 \mathrm{~b}$ illustrates this task for the projection only interaction technique. The task requires the user to select 4 of the 11 sights in the preferred order they would visit them. Selected sights were indicated using a purple border drawn around the sight icon. 


\subsection{Find a restaurant}

For the final task the user is required to enter a route comprising of a start and end point which was then displayed on the map (Figure 6c). Along the route several restaurant icons were placed. The user had to find and select the restaurant which was located at the center of the route. For this task when using the mobile phone and projector combination the projection displayed the map and the mobile phone permitted the use to enter start and end points in text format using the keypad.
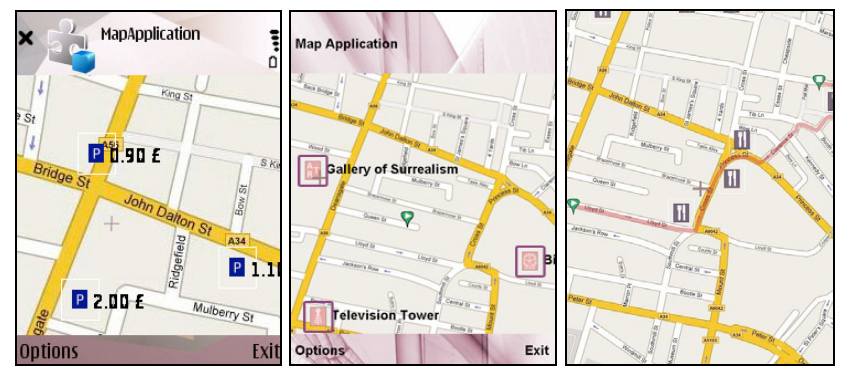

Figure 6. Map interaction tasks: (a) find cheapest car park, (b) sightseeing (c) find a restaurant.

As in the photo-browsing prototype the mobile phone screen was not used in the projection only modality. When using the combination of phone display and projection, the phone displayed menus and input fields for text input and the projection displayed the map. The left picture in Figure 7 shows the projection only version in which the map as well as the menu is displayed in the projection. The right picture in Figure 8 shows only the map as the menu is displayed on the phone using the corresponding modality.
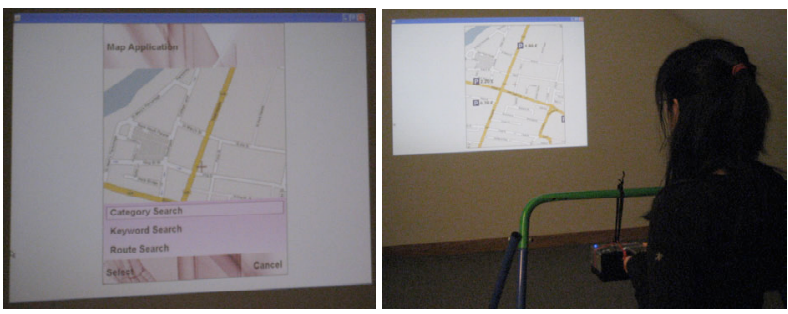

Figure 7. Interaction with the projector using projection only (left) and combination of projection and phone display (right).

\section{DISCUSSION}

There is the potential that with the ability to project anything, anyplace and practically any size will result in visual pollution [11]. It is clear that new privacy measures need to be designed and implemented to address this. It is also important to consider the brightness of the projector and the likely hood of being able to use a projector phone in a typical environment flooded by lights. The affects the projector imposes on battery life, social acceptability also needs to be considered. Microvision's 'Show' [12] is an accessory projector that can be connected to mobile devices has a brightness of 10 lumens, weighs $\sim 220$ grams and has a battery life of 1.5 hours. Finally, the physical position the projector mounted in the phone needs to be addressed. It may be the case that the current way in which we hold and interact with a mobile phone may make it difficult to use the projection or more so it may impact the ability to use the combination of the two displays.

\section{CONCLUSION}

In this paper we present a system that can be used for the design, development and evaluation of applications for projector phones as these devices are not yet available today. We show two different prototypes for photo browsing and map interaction as these are applications which benefit from a large high-resolution display as provided by a projector phone.

When showing the demonstration at Mobile HCI the attendees are able to use the two different applications, photo browsing and map interaction, and by doing so will see the distinct advantages and disadvantages and further issues to consider when using a projector phone.

\section{ACKNOWLEDGEMENT}

This work is supported by the NoE INTERMEDIA funded by the European Commission (NoE 038419).

\section{REFERENCES}

[1] Hang, A., Rukzio, E., Greaves, A. Projector Phone: A Study of Using Mobile Phones with Integrated Projector for Interaction with Maps. In Proceedings of Mobile HCI 2008. Amsterdam, Netherlands.

[2] Greaves, A., Rukzio, E. Picture Browsing and Map Interaction using a Projector Phone. In Proceedings of Mobile HCI 2008. Amsterdam, Netherlands.

[3] Chittaro, L. 2006. Visualizing Information on Mobile Devices. Computer (Mar., 2006), vol. 39, no. 3, 40-45.

[4] Baudisch, P. and Rosenholtz, R. 2003. Halo: A Technique for Visualizing Off-Screen Locations. In Proceedings of CHI 2003 (Fort Lauderdale, FL, April, 2003), 481-488.

[5] Cheverst, K., Dix, A., Fitton, D., Kray, C., Rouncefield, M., Sas, C., Saslis-Lagoudakis, G., and Sheridan, J. G. Exploring bluetooth based mobile phone interaction with the hermes photo display. MobileHCI 2005 (Salzburg, Austria, 2005).

[6] Greenberg, S., Boyle, M., Laberge, J. 1999. PDAs and shared public displays: Making personal information public, and public information personal. In Personal and Ubiquitous Computing, 3 (1-2), 54-64.

[7] Madden, D. CES 2008: DLP shows off working Pico projector prototype. 7.1.2008. http://www.pocketlint.co.uk/news/news.phtml/12082/13106/pico-projectorprototype-shown-off.phtml

[8] Meyer, D. Mobile-friendly projector debuts at CES. 7.1.2008. http://news.zdnet.co.uk/communications/ 0,1000000085,39291949,00.htm

[9] 3M Mobile Projection Technology http://www.3m.com/mpro/index.html

[10] Dredge, S. Nokia World: Fancy a 100GB mobile phone with a built-in projector? November 30, 2006. http://www.techdigest.tv/2006/11/nokia_world_fan.html

[11] Intrusion Warning over mini projectors. February 262008. http://news.bbc.co.uk/1/hi/technology/7265365.stm

[12] Microvision. SHOW Pico Projector Prototype. http://www.microvision.com/pdfs/show_specs.pdf 